\title{
Mild hypothermia protects against early brain injury in rats following subarachnoid hemorrhage via the TrkB/ERK/CREB signaling pathway
}

\author{
OU LV ${ }^{1}$, FENGGANG ZHOU ${ }^{2}$, YONGRI ZHENG ${ }^{2}$, QINGSONG LI ${ }^{2}$, JIANJIAO WANG ${ }^{2}$ and YULAN ZHU ${ }^{1}$ \\ Departments of ${ }^{1}$ Neurology and ${ }^{2}$ Neurosurgery, \\ The Second Affiliated Hospital of Harbin Medical University, Harbin, Heilongjiang 150086, P.R. China
}

Received August 5, 2015; Accepted August 18, 2016

DOI: $10.3892 / \mathrm{mmr} .2016 .5709$

\begin{abstract}
Subarachnoid hemorrhage (SAH) is a severe neurological disease, which is associated with a significant number of cases of premature mortality and disability worldwide. Mild hypothermia ( $\mathrm{MH}$ ) has been proposed as a potential therapeutic strategy to reduce neuronal injury following SAH. The present study aimed to investigate the mechanisms of MH's protective role in the process of SAH. The present study demonstrated that $\mathrm{MH}$ was able to protect against early brain injury in a rat model of SAH. Treating SAH rats with $\mathrm{MH}$ reduced the release of reactive oxygen species and prevented activation of apoptotic cascades. Furthermore, the protective effects of $\mathrm{MH}$ were shown to be mediated by enhanced activity of the tropomyosin receptor kinase B/extracellular signal-regulated kinases/cAMP response element binding protein (TrkB/ERK/CREB) pathway. Inhibition of TrkB/ERK/CREB activity using a small molecule inhibitor largely abolished the beneficial effects of $\mathrm{MH}$ in SAH rats. These results outline an endogenous mechanism underlying the neuroprotective effects of $\mathrm{MH}$ in $\mathrm{SAH}$.
\end{abstract}

\section{Introduction}

Subarachnoid hemorrhage $(\mathrm{SAH})$ is a severe neurological emergency, which accounts for $5 \%$ of all strokes (1). Although the diagnosis and treatment of SAH have improved in the past 20 years, SAH remains one of the most life-threatening acute neurological diseases, and is associated with a high rate of mortality and a poor prognosis. The primary cause of mortality in patients with SAH is brain injury that occurs during the early phase of SAH (within $48 \mathrm{~h}$ of SAH) (2), which

Correspondence to: Dr Yulan Zhu, Department of Neurology, The Second Affiliated Hospital of Harbin Medical University, 246 Xuefu Road, Nangang, Harbin, Heilongjiang 150086 P.R. China

E-mail: hydzhou@yeah.net

Key words: subarachnoid hemorrhage, mild hypothermia, TrkB/ERK/CREB is characterized by an initial sudden increase in intracranial pressure (ICP) and reduction in cerebral blood flow (CBF) (3). These complications trigger focal or global cerebral ischemia with various deleterious effects, including inflammation and neuronal cell death.

Due to the difficulties in predicting and preventing the occurrence of SAH, the management of SAH primarily focuses on protecting the brain from secondary damages during the acute and chronic phases that follow SAH. Mild hypothermia $(\mathrm{MH})$ is well known for its powerful neuroprotective effects against neuronal injury following ischemia and traumatic brain injury, including stroke (4). Several mechanisms have been proposed to underlie the protective effects of MH. For example, $\mathrm{MH}$ has been reported to reduce mitochondrial dysfunction and per-ischemic production of reactive oxygen species (ROS) following ischemic disorders, which is believed to be one of the major causes of cell death and inflammation post-ischemia $(5,6)$. Furthermore, MH treatment has been demonstrated to reduce global brain glucose and oxygen metabolic rates $(7,8)$, which may help the brain to deal with energy failure, and prevent mitochondrial dysfunction and neuronal apoptosis. Other studies have suggested that $\mathrm{MH}$ treatment stabilizes the blood-brain barrier (9), reduces brain edema (10) and the release of excitatory amino acids $(11,12)$, and attenuates inflammatory reactions (13) and lipid peroxidation (14).

Due to the well-documented protective effects of $\mathrm{MH}$ against ischemia and traumatic brain injury, $\mathrm{MH}$ has also been investigated as a potential therapeutic strategy for the treatment of SAH in humans and in animal models of SAH. Experimental studies in patients with $\mathrm{SAH}$ and animal models of SAH have suggested that $\mathrm{MH}$ treatment may improve ICP control (15), facilitate the resolution of cerebral vasospasm through modulation of blood flow velocity $(16,17)$, and prevent neuronal damage and apoptosis caused by cerebral ischemia. However, little is currently known regarding the molecular mechanisms and signaling pathways associated with the effects of $\mathrm{MH}$ on protection against early brain injury following $\mathrm{SAH}$.

Neurotrophic factors are required for the growth and survival of developing neurons and the maintenance of mature neurons. It has previously been reported that the tropomyosin receptor kinase B (TrkB)-mediated neurotrophic pathway has an important role in neuronal survival following ischemic 
stroke $(18,19)$. Pharmacological activation of TrkB-cAMP response element binding protein (CREB) may ameliorate ischemic neuronal injury via the prevention of neuronal apoptosis, and therefore may improve functional recovery following stroke $(18,19)$.

The present study aimed to determine the effects and mechanisms of $\mathrm{MH}$ on $\mathrm{SAH}$ development. The present study demonstrated that treatment with $\mathrm{MH}$ induced strong protective effects against neuronal injury in a rat model of SAH. Rats treated with $\mathrm{MH}$ exhibited a marked reduction in ROS production and caspase-3 activation following SAH. Furthermore, the TrkB/extracellular signal-regulated kinases (ERK)/CREB pathway mediated the protective effects of $\mathrm{MH}$. Suppression of the TrkB/ERK/CREB pathway using an ERK inhibitor markedly abrogated the protective effects of $\mathrm{MH}$ in SAH rats. These findings indicated that activation of the TrkB/ERK/CREB pathway may be an essential mechanism underlying the protective effects of $\mathrm{MH}$ against early brain injury following SAH in vivo.

\section{Materials and methods}

Animal study. All experiments were approved by the Animal Care Committee at Harbin Medical University (Harbin, China). Male Wistar rats (weight, 200-250 g; n=45) were housed in a temperature and humidity-controlled environment under a 12:12-h light/dark cycle (light phase, 7:00 a.m.-7:00 p.m.), and were given ad libitum access to food and water. The rat brain tissues were surgically removed following overdose with $5 \%$ isoflurane.

Rat model of SAH. The SAH model was established in male rats as described previously (20-22). Briefly, the rats were anesthetized. A sharpened 3-0 monofilament was introduced into the right internal carotid artery through the external carotid artery until resistance was felt (10-12 $\mathrm{mm}$ from the common carotid bifurcation). The monofilament was subsequently pushed further to perforate the bifurcation of the internal carotid artery, and was then withdrawn immediately. In the sham group, the monofilament was inserted into the carotid artery; however, no perforation was performed. Following removal of the monofilament, the incision was closed. Endovascular occlusion by perforation lasted $<5 \mathrm{~min}$ in each animal.

MH treatment. SAH was induced in 25 rats (except for 2 rats that had succumbed and the 3 which were excluded due to low weight, 30 rats purchased in total). Then, 5 rats were randomly selected and sacrificed at five different time points $(0,0.5,4,24$ and $72 \mathrm{~h})$ after induction.

A total of 20 rats (except for rats that had succumbed or did not qualify) were randomly divided into four groups: Sham, SAH, SAH + MH and SAH + MH + PD98059 groups. $\mathrm{MH}$ was conducted for $120 \mathrm{~min}$ as previously described (16), commencing $60 \mathrm{~min}$ after SAH. PD98059 $(5 \mathrm{mg} / \mathrm{kg} /$ day dissolved in $0.2 \mathrm{ml}$ dimethyl sulfoxide; New England Biolabs, Ipswich, MA, USA) was administered intravenously $0.5 \mathrm{~h}$ prior to SAH, in order to inhibit TrkB/ERK signaling as described previously (23). Mice were sacrificed 3 days after SAH for further evaluation.
Measurement of body weight and brain water content. The body weight and brain water content were measured on the third day following establishment of the SAH model. For brain water content, the brain tissues were removed, and the hemispheres were separated and weighed to assess their wet weight. After the wet weight of the brain tissues was quantified, the hemispheres were desiccated for $36 \mathrm{~h}$ at $110^{\circ} \mathrm{C}$, until the weight was constant. Hemispheric water content $(\%)$ was calculated as follows: (Wet weight - dried weight) / wet weight x $100 \%$.

ROS detection. Intracellular ROS levels were determined in brain tissue homogenates using 2',7'-dichlorodihydrofluorescein diacetate (DCFH-DA; Beyotime Institute of Biotechnology, Beijing, China) according to manufacturer's protocol. Briefly, the rat brain extracts were incubated with DCFH-DA at $37^{\circ} \mathrm{C}$ in the dark for $30 \mathrm{~min}$. The fluorescence intensity was then quantified using a multi-detection microplate reader at $485 \mathrm{~nm}$ excitation and $530 \mathrm{~nm}$ emission wavelengths.

Caspase-3 activity assay. Caspase-3 activity was determined using a CaspACE assay system (Promega Corporation, Madison, WI, USA) was conducted according to the manufacturer's protocol. Rat brain tissues were lysed on ice for $30 \mathrm{~min}$ and were centrifuged at $12,000 \times \mathrm{g}$ for $15 \mathrm{~min}$ at $4^{\circ} \mathrm{C}$. The levels of caspase- 3 were expressed relative to the amounts in the control group. The caspase- 3 activities in the supernatant were measured at $405 \mathrm{~nm}$.

Western blot analysis. The rat brains were surgically collected and cut into pieces. Subsequently, the brain tissues were directly homogenized in ice-cold lysis buffer [62.5 mM Tris- $\mathrm{HCl}, 2 \%$ (w/v) sodium dodecyl sulfate (SDS), 5\% (w/v) $\beta$-mercaptoethanol, $10 \%$ (v/v) glycerol, $0.002 \%$ (w/v) bromophenol blue] for $30 \mathrm{~min}$. The lysates were then centrifuged for $15 \mathrm{~min}$ at $12,000 \times \mathrm{g}$ at $4^{\circ} \mathrm{C}$, and the resulting supernatants were collected and boiled. Protein concentrations were measured in the extracts using a bichinchoninic acid assay. Protein samples (20-40 $\mu \mathrm{g} /$ lane) were separated by $10 \%$ SDS-polyacrylamide gel electrophoresis and were transferred to nitrocellulose membranes (Bio-Rad Laboratories, Inc., Hercules, CA, USA). The membranes were then blocked overnight with $5 \%$ bovine serum albumin (Sigma-Aldrich; Merck Millipore, Darmstadt, Germany) in Tris-buffered saline containing $0.1 \%$ Tween-20 (TBST), and were incubated with antibodies against cleaved caspase-3 (cat. no. ab2302; 1:1,000; Abcam, Cambridge, UK), phosphorylated (p)-TrkB (sc-7987, 1: 500; Santa Cruz Biotechnology, Inc., Dallas, TX, USA), TrkB (cat. no. sc-377218; 1:1,000; Santa Cruz Biotechnology, Inc.), p-ERK1/2 (cat. no. sc-136521; 1:1,000; Santa Cruz Biotechnology,Inc.),ERK1/2 (cat.no. sc-292838; 1:1,000; Santa Cruz Biotechnology, Inc.) p-CREB (cat. no. sc-7978, 1:1,000; Santa Cruz Biotechnology, Inc.), CREB (cat. no. sc-377154; 1:1,000; Santa Cruz Biotechnology, Inc.) and glyceraldehyde 3-phosphate dehydrogenase (GAPDH; cat. no. sc-47724; 1:1,000; Santa Cruz Biotechnology, Inc.) at $4^{\circ} \mathrm{C}$ overnight, followed by washing with TBST. The membranes were then incubated for $2 \mathrm{~h}$ at room temperature with horseradish peroxidase-conjugated secondary antibody (Wuhan Boster Biological Technology Co., Ltd., Wuhan, China). The blots were detected using an Enhanced Chemiluminescence Plus 
A

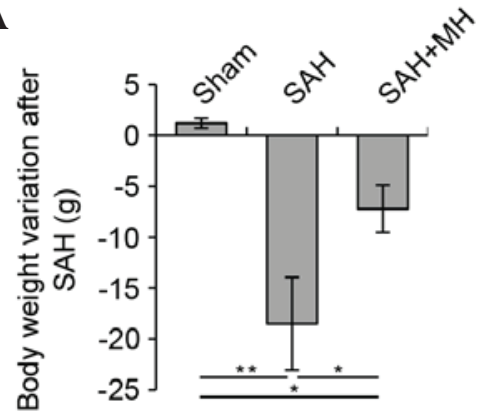

D

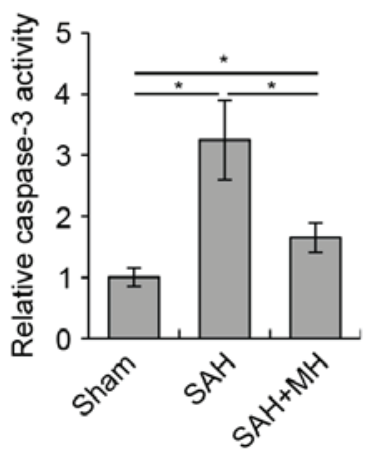

B

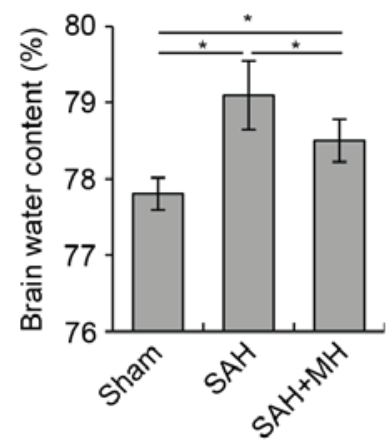

E

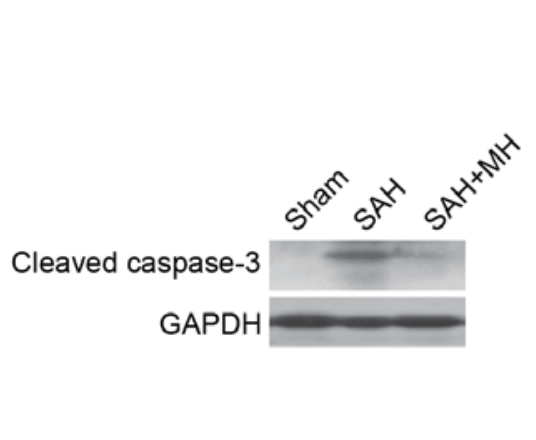

C
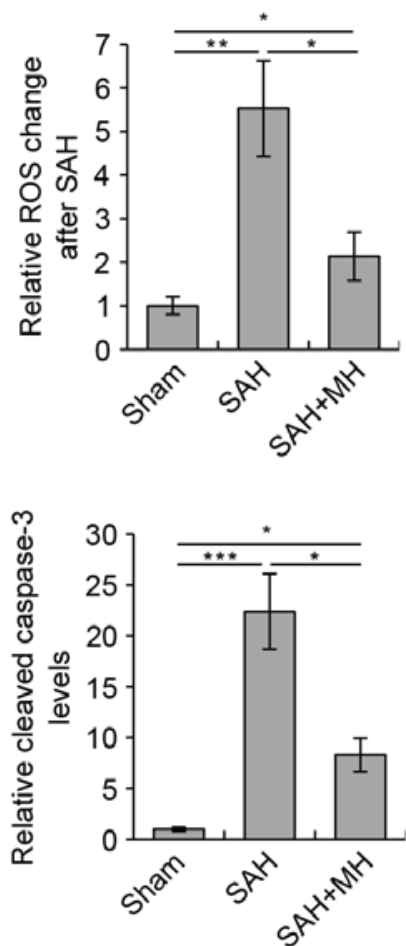

Figure 1. Mild hypothermia (MH) protects against early brain injury in a rat model of subarachnoid hemorrhage (SAH). (A) Body weight of rats in sham, SAH and $\mathrm{SAH}+\mathrm{MH}$ groups 3 days after SAH. (B) Brain water content, (C) relative reactive oxygen species (ROS) release and (D) relative caspase-3 activity in sham, $\mathrm{SAH}$ and $\mathrm{SAH}+\mathrm{MH}$ groups 3 days after $\mathrm{SAH}$. (E) Western blot analysis of cleaved caspase-3 and quantification (right). ${ }^{*} \mathrm{P}<0.05 ;{ }^{* *} \mathrm{P}<0.01 ;{ }^{* * *} \mathrm{P}<0.001$. GAPDH, glyceraldehyde 3-phosphate dehydrogenase.

reagent kit (Wuhan Boster Biological Technology Co., Ltd.). GAPDH was used as a loading control. The bands were quantified using Image J version 1.37 software (National Institutes of Health, Bethesda, MD, USA).

Immunofluorescence staining. The brain tissues were fixed in $4 \%$ paraformaldehyde, and were sliced using a cryostat. The sections $(20 \mu \mathrm{m})$ were stored in anti-freeze solution (15\% glucose and $30 \%$ ethylene glycol in $50 \mathrm{mM}$ phosphate buffer, pH 7.4) at $-20^{\circ} \mathrm{C}$ and were used for immunofluorescence staining. Frozen sections were incubated overnight at $4^{\circ} \mathrm{C}$ with primary antibodies against cleaved caspase-3 (cat.no. ab2302; 1:100; Abcam) and p-CREB (cat. no. sc-7987; 1:100; Santa Cruz Biotechnology, Inc.). The slices were then washed three times with phosphate-buffered saline and were incubated with the corresponding fluorescence dye-conjugated bovine anti-rabbit IgG-FITC (cat. no. sc-2365; 1:100; Santa Cruz Biotechnology, Inc.) and donkey anti-goat IgG-TR (cat. no. sc-2783; 1:100; Santa Cruz Biotechnology, Inc.) secondary antibodies for $2 \mathrm{~h}$. After being washed and counterstained with 4',6-diamidino-2-phenylindole, immunofluorescence was observed under a fluorescence microscope (Olympus Corporation, Tokyo, Japan).

Statistical analysis. All data are presented as the mean \pm standard error of the mean. Group differences were analyzed using one-way analysis of variance followed by Tukey's honest significant difference test. Statistical analyses were conducted using GraphPad Prism version 5.0 statistical software
(GraphPad Software, Inc., La Jolla, CA, USA). P<0.05 was considered to indicate a statistically significant difference.

\section{Results}

$M H$ protects against early brain injury in rats following $S A H$. To investigate the protective function of $\mathrm{MH}$ in $\mathrm{SAH}$, a rat model of SAH was generated, and the rats were treated with $\mathrm{MH} 1 \mathrm{~h}$ after the model was established. Consistent with previous observations regarding this model (20-22), the induction of SAH reduced body weight and increased brain water content in the rats. These detrimental effects caused by $\mathrm{SAH}$ were significantly attenuated by $\mathrm{MH}$, as indicated by the reduction in body weight loss (Fig. 1A) and brain water accumulation (Fig. 1B). Given that mitochondrial dysfunction and the activation of apoptotic cascades are key pathological events in early brain injury following SAH $(24,25)$, the present study investigated whether $\mathrm{MH}$ was able to protect neurons from mitochondrial dysfunction and apoptosis in early brain injury. The results clearly demonstrated that $\mathrm{MH}$ improved mitochondrial function following $\mathrm{SAH}$, as evidenced by the reduction in ROS production (Fig. 1C). Furthermore, the activation of caspase-3 was markedly reduced by $\mathrm{MH}$ (Fig. 1D and $\mathrm{E}$ ). These data indicate that $\mathrm{MH}$ treatment in the early phase of SAH may reduce ROS release and neuronal apoptosis, thus improving the outcome of $\mathrm{SAH}$ in vivo.

$M H$ promotes TrkB/ERK/CREB signaling. The present study aimed to determine the molecular mechanism underlying 
A
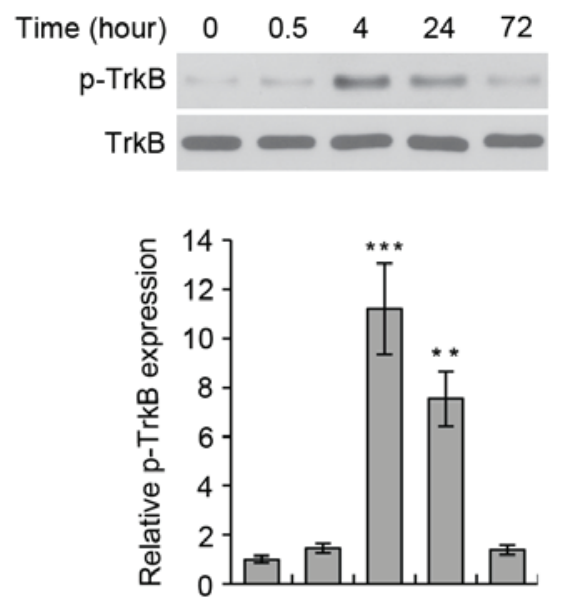

Time (hour) $0 \quad 0.5 \quad 4 \quad 24 \quad 72$
B
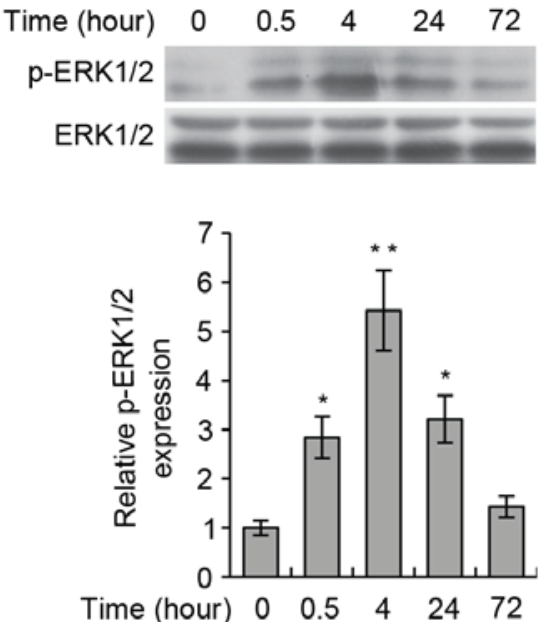

C
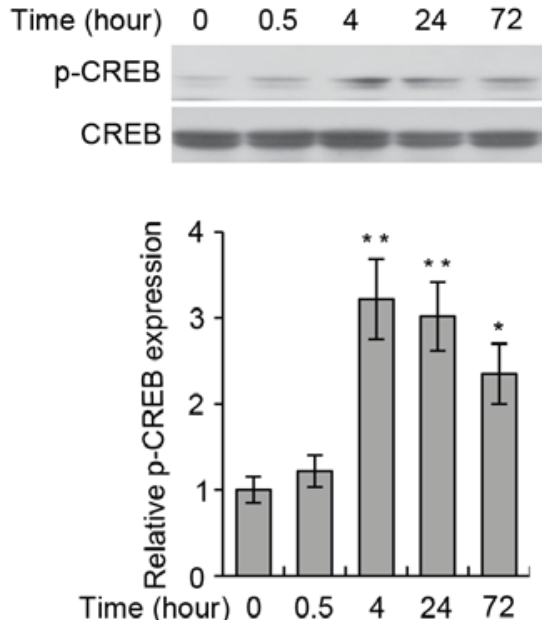
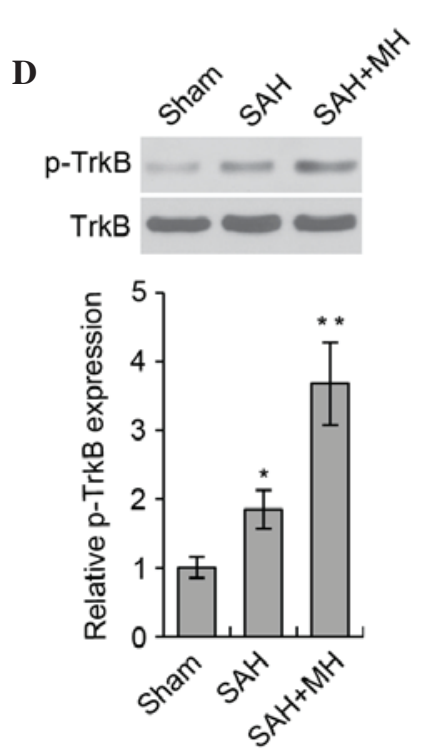

E
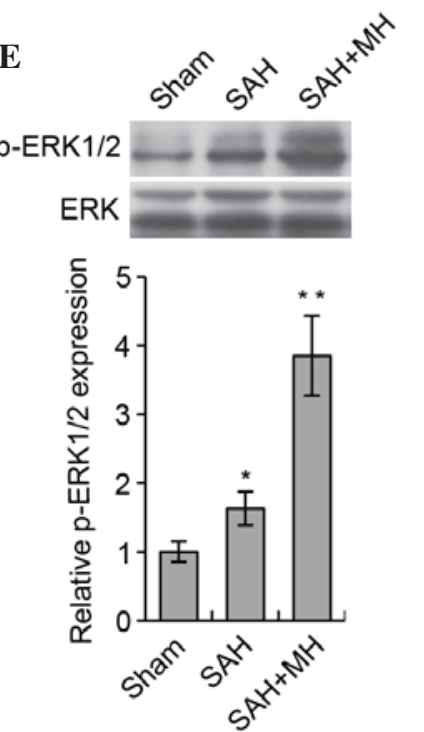

F
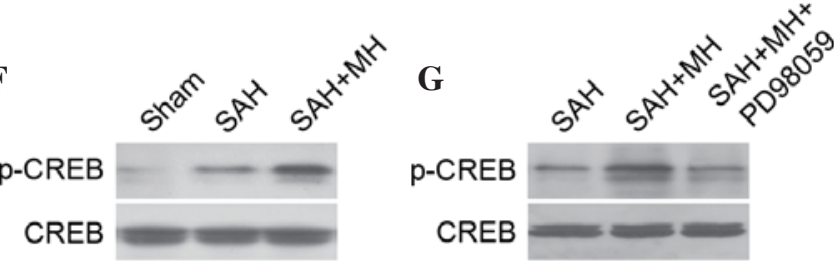

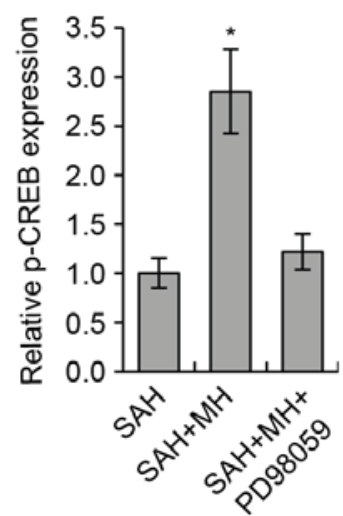

Figure 2. Mild hypothermia (MH) promotes activation of the tropomyosin receptor kinase B/extracellular signal-regulated kinases/cAMP response element binding protein (TrkB/ERK/CREB) pathway. Western blot analysis of (A) phosphorylated (p)-TrkB, (B) p-ERK1/2 and (C) p-CREB expression in rat brains following induction of subarachnoid hemorrhage (SAH), at various time points $(0,0.5,4,24$ and $72 \mathrm{~h})$. Western blot analysis of (D) p-TrkB, (E) p-ERK1/2 and (F) p-CREB expression in rat brains $72 \mathrm{~h}$ post-SAH induction, with or without $\mathrm{MH}$ treatment. (G) p-CREB expression in rat brains following SAH, and treatment with $\mathrm{MH}$ or $\mathrm{MH}+\mathrm{PD} 98059 .{ }^{*} \mathrm{P}<0.05 ;{ }^{* *} \mathrm{P}<0.01 ;{ }^{* * *} \mathrm{P}<0.001$ vs. sham or $0 \mathrm{~h}$ group.

the protective effects of $\mathrm{MH}$ on SAH. The TrkB-mediated neurotrophic pathway has critical roles in neuronal survival and growth. Previous studies have demonstrated that $\mathrm{MH}$ was able to induce the expression of brain-derived neurotrophic factor (BDNF) in rat brains following cerebral ischemia; BDNF functions as a ligand of the TrkB receptor $(26,27)$. Therefore, the present study hypothesized that $\mathrm{MH}$ would prevent neuronal injury via activation of the TrkB-mediated neurotrophic pathway. The activity of TrkB, and the downstream ERK1/2/CREB pathway, was analyzed at various time points $(0,0.5,4,24$ and $72 \mathrm{~h})$ following SAH. SAH markedly stimulated TrkB/ERK/CREB signaling (Fig. 2A-C). The levels of p-TrkB, p-ERK1/2 and p-CREB in the rat brain peaked at $4 \mathrm{~h}$, and then decreased gradually. Treatment with MH markedly enhanced the phosphorylation of TrkB (Fig. 2D), ERK1/2 (Fig. 2E) and CREB (Fig. 2F) following SAH. Furthermore, $\mathrm{MH}$-induced phosphorylation of CREB was shown to be dependent on the activation of TrkB and ERK, since inhibition of ERK using the small molecule inhibitor PD98059 markedly abrogated CREB phosphorylation (Fig. 2G). These data indicate that the TrkB/ERK/CREB signaling pathway may be involved in the progress of $\mathrm{SAH}$, and that $\mathrm{MH}$ promotes activation of the TrkB/ERK/CREB pathway following SAH.

Inhibition of the TrkB/ERK/CREB signaling pathway reduces the protective effects of $M H$ in $S A H$-induced early brain injury. To examine whether the TrkB/ERK/CREB signaling pathway is required for the protective effects of $\mathrm{MH}$, the effects of $\mathrm{MH}$ on early brain injury were detected following inhibition of the TrkB/ERK/CREB pathway using PD98059. Notably, inhibition of the TrkB/ERK/CREB pathway almost completely abrogated the beneficial effects of $\mathrm{MH}$ on $\mathrm{SAH}$. The reductions in body weight loss and brain water content were reversed by PD98059. There was no significant difference in body weight 


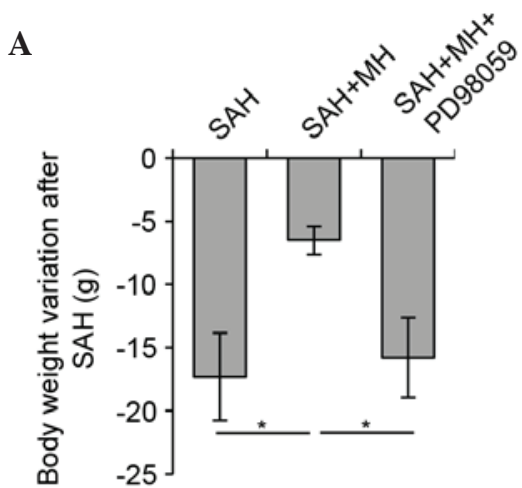

D

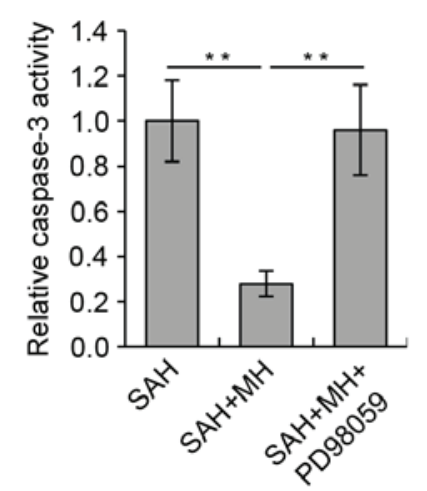

$\mathbf{B}$

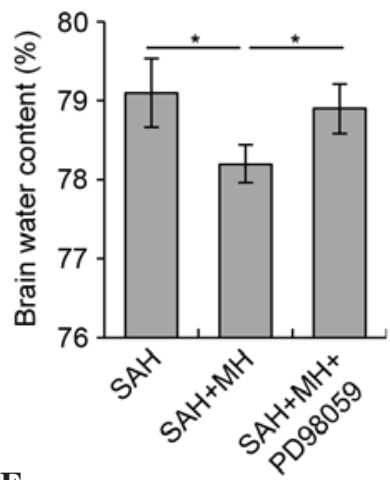

$\mathbf{E}$

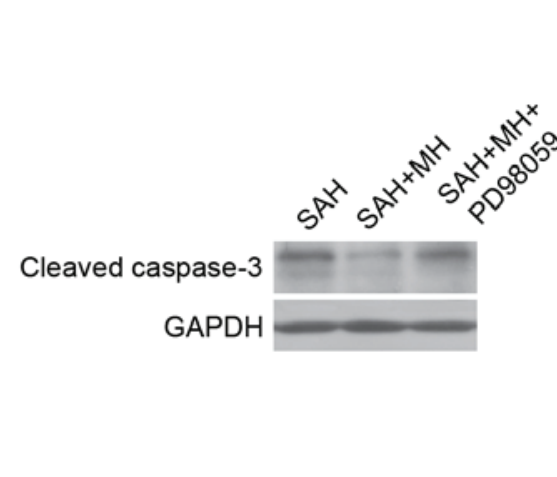

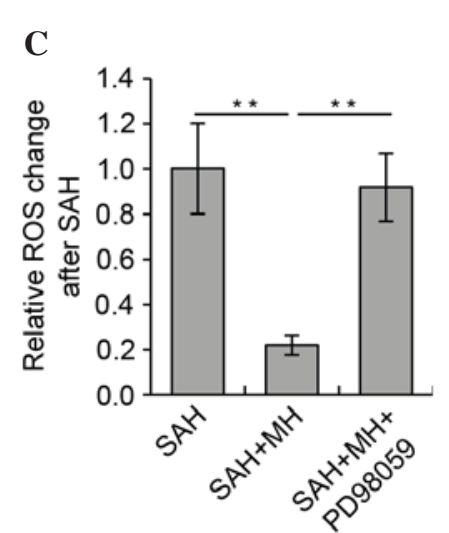

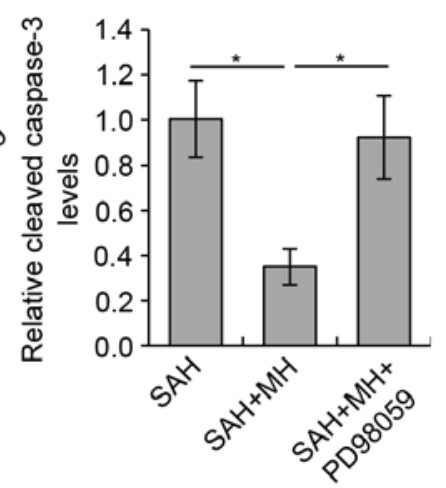

Figure 3. Inhibition of the tropomyosin receptor kinase B/extracellular signal-regulated kinases/cAMP response element binding protein (TrkB/ERK/CREB) pathway reduces the protective effects of mild hypothermia $(\mathrm{MH})$ on early brain injury following subarachnoid hemorrhage (SAH). (A) Body weight of SAH rats treated with $\mathrm{MH}$ or $\mathrm{MH}+\mathrm{PD} 98059$. (B) Brain water content, (C) relative reactive oxygen species (ROS) release and (D) relative caspase-3 activity in the brains of SAH rats treated with MH or MH + PD98059. (E) Western blot analysis of cleaved caspase-3 levels and quantification (right). "P<0.05; ${ }^{* *} \mathrm{P}<0.01$. GAPDH, glyceraldehyde 3-phosphate dehydrogenase.

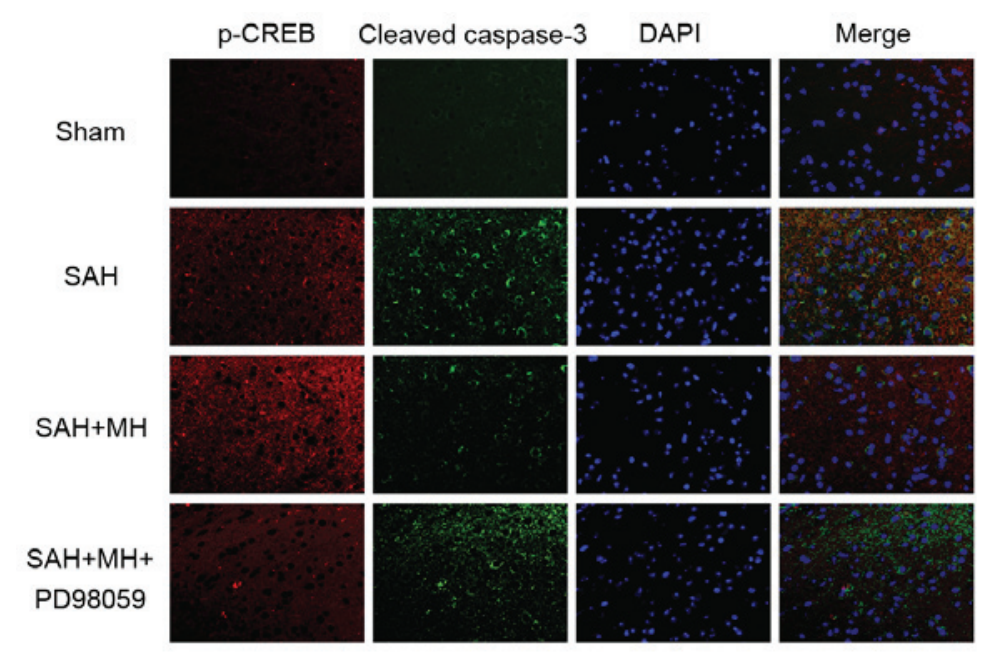

Figure 4. Immunohistochemical staining of phosphorylated-cAMP response element binding protein (p-CREB) and cleaved caspase-3. Immunofluorescent staining of p-CREB (red) and cleaved caspase-3 (green) in rat brain sections; 4',6-diamidino-2-phenylindole (DAPI; blue) was used for nuclear staining. Magnification, $\mathrm{x}$ 400. SAH, subarachnoid hemorrhage; $\mathrm{MH}$, mild hypothermia.

(Fig. 3A) and brain water content (Fig. 3B) between the SAH and $\mathrm{SAH}+\mathrm{MH}+\mathrm{PD} 98059$ groups. Treatment with PD98059 also abrogated the effects of $\mathrm{MH}$ on the prevention of ROS production (Fig. 3C) and caspase-3 activation (Fig. 3D) following $\mathrm{SAH}$.

To determine the role of CREB in SAH and MH-treated $\mathrm{SAH}$, fluorescence staining of $\mathrm{p}-\mathrm{CREB}$ and cleaved caspase-3 was performed on brain sections. SAH induced extensive cell apoptosis and promoted CREB phosphorylation (Fig. 4). In addition, $\mathrm{MH}$ increased the levels of $\mathrm{p}-\mathrm{CREB}$, and decreased the levels of cleaved caspase-3. These effects were abrogated by inhibition of TrkB/ERK signaling. These results indicate that $\mathrm{MH}$ may protect the brain from $\mathrm{SAH}$-induced neuronal injury by activating the TrkB/ERK/CREB signaling pathway. 


\section{Discussion}

MH has been revealed to be effective in minimizing neuronal damage and improving the functional outcome of SAH; however, the molecular mechanisms underlying the beneficial effects of MG remain unclear. Using a rat SAH perforation model, the present study demonstrated that MH was able to attenuate mitochondrial dysfunction and activation of apoptosis in the brain following the induction of SAH. These protective effects were mediated by enhanced activity of the TrkB/ERK/CREB pathway. These observations identify a potential mechanism by which $\mathrm{MH}$ protects rats from early brain damage following $\mathrm{SAH}$.

Chronic post-SAH pathological consequences, characterized by delayed cerebral ischemia and vasospasm of the major cerebral arteries (3-7 days after SAH), have been extensively studied and treated; however, these efforts have not resulted in an effective treatment to prevent or ameliorate brain injury following SAH $(28,29)$. The early brain injury that occurs within $48 \mathrm{~h}$ of SAH has gained more attention as a novel target for improving SAH patient outcome, since $>60 \%$ of patients with SAH succumb due to early brain injury during the first $48 \mathrm{~h}$ after SAH $(30,31)$. In addition, the majority of chronic secondary injuries are initiated by early brain injury. It has previously been indicated that mitochondrial dysfunction and extensive neuronal apoptosis are the key events associated with early brain injury following SAH (32). Following $\mathrm{SAH}$-induced global ischemia, apoptosis has been observed in several regions of the brain, including the hippocampus, blood-brain barrier (BBB) and vasculature (24). Activation of apoptotic cascades may lead to severe pathological complications, including BBB disruption (33) and vasospasm (34). Therefore, anti-apoptosis may be considered a potential therapeutic intervention for the treatment of SAH.

MH is a neuroprotective approach that has been employed in various clinical scenarios, particularly in the treatment of ischemic stroke and traumatic brain injury. Based on the pathophysiological similarity between stroke and SAH, MH has been tested as a potential therapy for SAH in humans and animal models of SAH. Several studies have indicated that $\mathrm{MH}$ may reduce ICP and improve CBF in the early phase of $\mathrm{SAH}$, and minimize the detrimental effects of delayed cerebral ischemia and vasospasm in the chronic secondary injuries following $\mathrm{SAH}$, thus improving the functional outcome in patients with SAH $(35,36)$. Although the exact molecular mechanisms underlying the protective effects of $\mathrm{MH}$ on $\mathrm{SAH}$ are largely unknown, it is generally believed that $\mathrm{MH}$ protects against neuronal damage via several mechanisms. The present study demonstrated that $\mathrm{MH}$ reduced ROS generation and activation of apoptotic cascades during the early phase following $\mathrm{SAH}$. These results suggested that MH may reduce neuronal damage, at least partially, through improving mitochondrial function and promoting neuronal survival.

BDNF/TrkB signaling is critical for neuronal survival, morphogenesis and plasticity. It is well-known that activation of the TrkB receptor elicits various intracellular signaling pathways, including the mitogen-activated protein kinase/ERK pathway, the phosphoinositide 3-kinase pathway, and CREB transcription (37). All of these pathways have been reported to participate in the regulation of neuronal growth and survival (37). Beyond its physiological function, pharmaceutical activation of the TrkB pathway promotes neuronal survival following ischemic brain injury $(19,38)$. The present study established a previously unappreciated link between $\mathrm{MH}$ and the neurotrophic pathway. MH enhanced activity of the TrkB/ERK/CREB pathway in vivo in a rat model of SAH. Notably, the TrkB/ERK/CREB pathway is essential for the neuroprotective effects of $\mathrm{MH}$ on $\mathrm{SAH}$, since inhibition of this pathway using a small molecule inhibitor almost fully abolished the beneficial effects of $\mathrm{MH}$. These results indicated a novel protective mechanism for $\mathrm{MH}$ in the context of early brain injury following SAH. Based on these findings, MH may reduce neuronal loss not only through inhibiting cell death activators (such as c-Jun N-terminal kinase) (39) but also through enhancing pro-survival signaling pathways. Notably, studies in other animal models of ischemic brain injury have suggested that treatment with $\mathrm{MH}$ could induce BDNF expression in the hippocampus $(26,27)$, which may be a potential mechanism that explains how $\mathrm{MH}$ treatment activates the TrkB/ERK/CREB pathway. The phosphorylated TrkB, ERK and CREB were stumilated by SAH and reached peak at $4 \mathrm{~h}$ after hemorrhage. The levels of phosphorylated protein then decreased due to short half-lives. Downstream target genes were then elevated (40-42).

In conclusion, using a rat model of $\mathrm{SAH}$, the present study demonstrated that $\mathrm{MH}$ ameliorates early brain injury through the prevention of mitochondrial dysfunction and inhibition of apoptotic cascades following SAH. The beneficial effects of $\mathrm{MH}$ are largely dependent on activation of the TrkB/ERK/CREB pathway. In the past few decades, early brain injury has evolved to be a promising therapeutic target for SAH. The present study indicated that $\mathrm{MH}$ is an effective strategy that may be used to reduce neuronal damage in the early phase of SAH. This mechanistic study of $\mathrm{MH}$ action in SAH revealed that the TrkB/ERK/CREB pathway may represent a novel therapeutic target for the intervention of early brain injury following SAH.

\section{Acknowledgements}

The present study was supported by the Postdoctoral Grant of Harbin Medical University (grant no. 2014M561373).

\section{References}

1. van Gijn J, Kerr RS and Rinkel GJ: Subarachnoid haemorrhage. Lancet 369: 306-318, 2007.

2. Ostrowski RP, Colohan AR and Zhang JH: Molecular mechanisms of early brain injury after subarachnoid hemorrhage. Neurol Res 28: 399-414, 2006.

3. Bederson JB, Levy AL, Ding WH, Kahn R, DiPerna CA, Jenkins AL III and Vallabhajosyula P: Acute vasoconstriction after subarachnoid hemorrhage. Neurosurgery 42: 352-362, 1998.

4. Thome C, Schubert GA and Schilling L: Hypothermia as a neuroprotective strategy in subarachnoid hemorrhage: A pathophysiological review focusing on the acute phase. Neurol Res 27: 229-237, 2005.

5. Tissier R, Chenoune M, Pons S, Zini R, Darbera L, Lidouren F, Ghaleh B, Berdeaux A and Morin D: Mild hypothermia reduces per-ischemic reactive oxygen species production and preserves mitochondrial respiratory complexes. Resuscitation 84: 249-255, 2013.

6. Lee SM, Zhao H, Maier CM and Steinberg GK: The protective effect of early hypothermia on PTEN phosphorylation correlates with free radical inhibition in rat stroke. J Cereb Blood Flow Metab 29: 1589-1600, 2009. 
7. Milde LN: Clinical use of mild hypothermia for brain protection: A dream revisited. J Neurosurg Anesthesiol 4: 211-215, 1992.

8. Erecinska M, Thoresen M and Silver IA: Effects of hypothermia on energy metabolism in Mammalian central nervous system. J Cereb Blood Flow Metab 23: 513-530, 2003.

9. Smith SL and Hall ED: Mild pre- and posttraumatic hypothermia attenuates blood-brain barrier damage following controlled cortical impact injury in the rat. J Neurotrauma 13: 1-9, 1996.

10. Karibe H, Zarow GJ, Graham SH and Weinstein PR: Mild intraischemic hypothermia reduces postischemic hyperperfusion, delayed postischemic hypoperfusion, blood-brain barrier disruption, brain edema, and neuronal damage volume after temporary focal cerebral ischemia in rats. J Cereb Blood Flow Metab 14: 620-627, 1994

11. Maeda T, Katayama Y,Kawamata T and Yamamoto T: Mechanisms of excitatory amino acid release in contused brain tissue: Effects of hypothermia and in situ administration of $\mathrm{Co} 2+$ on extracellular levels of glutamate. J Neurotrauma 15: 655-664, 1998.

12. Mori K, Maeda M, Miyazaki M and Iwase H: Effects of mild and moderate hypothermia on cerebral metabolism and glutamate in an experimental head injury. Acta Neurochir Suppl 71: 222-224, 1998

13. Chatzipanteli K, Yanagawa Y, Marcillo AE, Kraydieh S, Yezierski RP and Dietrich WD: Posttraumatic hypothermia reduces polymorphonuclear leukocyte accumulation following spinal cord injury in rats. J Neurotrauma 17: 321-332, 2000.

14. Busto R, Globus MY, Dietrich WD, Martinez E, Valdes I and Ginsberg MD: Effect of mild hypothermia on ischemia-induced release of neurotransmitters and free fatty acids in rat brain. Stroke 20: 904-910, 1989

15. Gasser S, Khan N, Yonekawa Y, Imhof HG and Keller E: Long-term hypothermia in patients with severe brain edema after poor-grade subarachnoid hemorrhage: Feasibility and intensive care complications. J Neurosurg Anesthesiol 15: 240-248, 2003.

16. Thome C, Schubert G, Piepgras A, Elste V, Schilling L and Schmiedek P: Hypothermia reduces acute vasospasm following SAH in rats. Acta Neurochir Suppl 77: 255-258, 2001.

17. Bishop CC, Powell S, Rutt D and Browse NL: Transcranial Doppler measurement of middle cerebral artery blood flow velocity: A validation study. Stroke 17: 913-915, 1986.

18. Hasegawa Y, Suzuki H, Altay O and Zhang JH: Preservation of tropomyosin-related kinase B (TrkB) signaling by sodium orthovanadate attenuates early brain injury after subarachnoid hemorrhage in rats. Stroke 42: 477-483, 2011.

19. He Q, Wang S, Liu X, Guo H, Yang H, Zhang L, Zhuang P, Zhang Y, Ye Z and Hu L: Salvianolate lyophilized injection promotes post-stroke functional recovery via the activation of VEGF and BDNF-TrkB-CREB signaling pathway. Int J Clin Exp Med 8: 108-122, 2015.

20. Doczi T, Laszlo FA, Szerdahelyi P and Joo F: Involvement of vasopressin in brain edema formation: Further evidence obtained from the brattleboro diabetes insipidus rat with experimental subarachnoid hemorrhage. Neurosurgery 14: 436-441, 1984.

21. Piepgras A, Elste V, Frietsch T, Schmiedek P, Reith W and Schilling L: Effect of moderate hypothermia on experimental severe subarachnoid hemorrhage, as evaluated by apparent diffusion coefficient changes. Neurosurgery 48: 1128-1135, 2001

22. Sun BL, Zhang SM, Xia ZL, Yang MF, Yuan H, Zhang J and Xiu RJ: The effects of nimodipine on regional cerebral blood flow, brain water and electrolyte contents in rats with subarachnoid hemorrhage. Clin Hemorheol Microcirc 29: 337-344, 2003.

23. Wojcicka G, Jamroz-Wisniewska A, Widomska S, Ksiazek M and Bełtowski J: Role of extracellular signal-regulated kinases (ERK) in leptin-induced hypertension. Life Sci 82: 402-412, 2008.

24. Cahill J, Calvert JW and Zhang JH: Mechanisms of early brain injury after subarachnoid hemorrhage. J Cereb Blood Flow Metab 26: 1341-1353, 2006
25. Niizuma K, Endo $\mathrm{H}$ and Chan PH: Oxidative stress and mitochondrial dysfunction as determinants of ischemic neuronal death and survival. J Neurochem 109 (Suppl 1): S133-S138, 2009.

26. D'Cruz BJ, Fertig KC, Filiano AJ, Hicks SD, DeFranco DB and Callaway CW: Hypothermic reperfusion after cardiac arrest augments brain-derived neurotrophic factor activation. J Cereb Blood Flow Metab 22: 843-851, 2002.

27. Boris-Moller F, Kamme F and Wieloch T: The effect of hypothermia on the expression of neurotrophin mRNA in the hippocampus following transient cerebral ischemia in the rat. Brain Res Mol Brain Res 63: 163-173, 1998.

28. McGirt MJ, Garces Ambrossi GL, Huang J and Tamargo RJ: Simvastatin for the prevention of symptomatic cerebral vasospasm following aneurysmal subarachnoid hemorrhage: A single-institution prospective cohort study. J Neurosurg 110: 968-974, 2009.

29. Vajkoczy P, Meyer B, Weidauer S, Raabe A, Thome C, Ringel F, Breu V and Schmiedek P: Clazosentan (AXV-034343), a selective endothelin A receptor antagonist, in the prevention of cerebral vasospasm following severe aneurysmal subarachnoid hemorrhage: Results of a randomized, double-blind, placebo-controlled, multicenter phase IIa study. J Neurosurg 103: 9-17, 2005.

30. Broderick JP, Brott TG, Duldner JE, Tomsick T and Leach A: Initial and recurrent bleeding are the major causes of death following subarachnoid hemorrhage. Stroke 25: 1342-1347, 1994.

31. Le Roux PD and Winn HR: Management of the ruptured aneurysm. Neurosurg Clin N Am 9: 525-540, 1998.

32. Abe Y, Sakairi T, Kajiyama H, Shrivastav S, Beeson C and Kopp JB: Bioenergetic characterization of mouse podocytes. Am J Physiol 299: 464-476, 2010.

33. Germanò A, d'Avella D, Imperatore C, Caruso $G$ and Tomasello F: Time-course of blood-brain barrier permeability changes after experimental subarachnoid haemorrhage. Acta Neurochir (Wien) 142: 575-581, 2000.

34. Zhou C, Yamaguchi M, Colohan AR and Zhang JH: Role of p53 and apoptosis in cerebral vasospasm after experimental subarachnoid hemorrhage. J Cereb Blood Flow Metab 25: $572-582,2005$

35. Kirkman MA and Smith M: Intracranial pressure monitoring, cerebral perfusion pressure estimation, and ICP/CPP-guided therapy: A standard of care or optional extra after brain injury? Br J Anaesth 112: 35-46, 2014.

36. Haddad SH and Arabi YM: Critical care management of severe traumatic brain injury in adults. Scand J Trauma Resusc Emerg Med 20: 12, 2012.

37. Numakawa T, Suzuki S, Kumamaru E, Adachi N, Richards M and Kunugi $\mathrm{H}$ : BDNF function and intracellular signaling in neurons. Histol Histopathol 25: 237-258, 2010.

38. Tian X, Guo J, Zhu M, Li M, Wu G and Xia Y: $\delta$-Opioid receptor activation rescues the functional TrkB receptor and protects the brain from ischemia-reperfusion injury in the rat. PLoS One 8: e69252, 2013

39. Xu L, Yenari MA, Steinberg GK and Giffard RG: Mild hypothermia reduces apoptosis of mouse neurons in vitro early in the cascade. J Cereb Blood Flow Metab 22: 21-28, 2002.

40. Fujioka A, Terai K, Itoh RE, Aoki K, Nakamura T, Kuroda S, Nishida E and Matsuda M: Dynamics of the Ras/ERK MAPK cascade as monitored by fluorescent probes. J Biol Chem 281: 8917-8926, 2006

41. Yang T, Massa SM and Longo FM: LAR protein tyrosine phosphatase receptor associates with TrkB and modulates neurotrophic signaling pathways. J Neurobiol 66: 1420-1436, 2006.

42. Sánchez-Huertas C and Rico B: CREB-dependent regulation of GAD65 transcription by BDNF/TrkB in cortical interneurons. Cereb Cortex 21: 777-788, 2011. 